\title{
WIRELESS SENSOR NETWORKS COMMUNICATION: ENERGY EFFICIENT PARADIGM
}

\author{
Roopashree $^{1}$, Gouri Patil ${ }^{2}$, Pradeepkumar Patil ${ }^{3}$ \\ ${ }^{I} P G$ Student Department of CNE, Appa Institute of engineering and technology, Gulbarga, Karnataka, India \\ ${ }^{2} P G$ Student Department of CNE, Appa Institute of engineering and technology, Gulbarga, Karnataka, India \\ ${ }^{3}$ Asst. Prof. Department of CSE, Appa Institute of engineering and technology, Gulbarga, Karnataka, India
}

\begin{abstract}
As the number of application in wireless sensor networks are increasing because of ongoing miniaturization of electro-mechanical parts, its subsidiary elements and drastic decrease in the cost. The communication among sensor nodes consumes more energy than the actual computation of information. The consumption not only takes place in transmission state, but also relatively high in idle state. The focus of this paper is to identify the main source of energy dissipation, as well as counter measure to ensure prolonged network lifetime. In addition to these, different types of protocols for routing, medium access control and transport protocols are presented and clarified by the means of examples.
\end{abstract}

Keywords: Energy conservation, energy efficient communication.

\section{INTRODUCTION}

Wireless sensor network's is growing tremendously because of ongoing miniaturization of electro-mechanical parts and decrease in their cost for manufacturing. The sensing device and the methods opted for measuring various phenomenons. Due to the lack of (energy) resource of the sensor nodes, the targeted approaches are required to make the demands for long running networks. Usually most of the energy is consumed at the source end, data processing and communication. The basis for identifying and exploiting the energy saving protocols are presented. Typically a large number of sensor nodes is randomly deployed within a geographic area. The basic functionality of those sensor nodes is data sensing, data processing and communication with other nodes or base stations. To perform these operations the sensor nodes need to have a power supply - usually a battery, it serves as a one-way product and cannot be changed. As sensor nodes are both data originator and data router [17], hence they collaborate to deliver data from source to sink. [15] Distinguishes between terminal nodes which only collect data and the in addition to this data forwarding intermediate nodes. Furthermore the sensor nodes can be consolidated in distinct clusters with a certain cluster head where the sensed data of direct neighbours, which is most likely to have some consonances, is brought together prior to the transmission.

All sensing information from the actual sensor nodes is gathered at base stations, whose energy resources are not as limited as the node's energy storage, since they are commonly equipped with a permanent connection to a continual power supply.

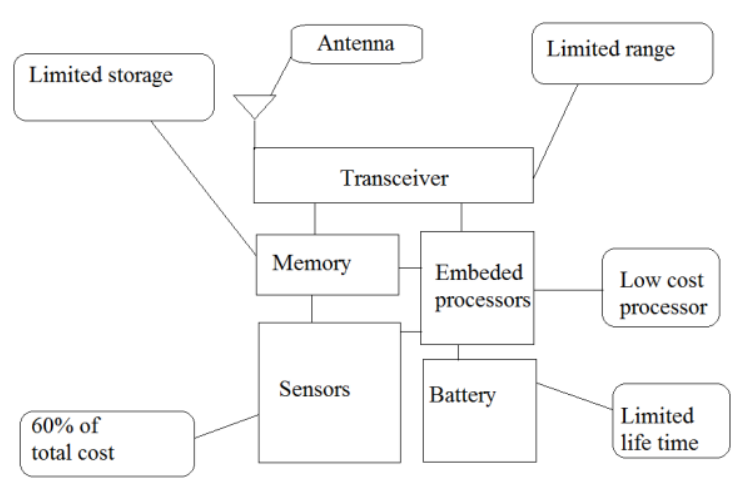

Fig 1: A basic set up of sensor node.

At those base stations the transmitted data from the sensor nodes is processed and the results are forwarded to the point of delivery where the user can access the collective sensing yield.

\section{DESIGN}

As stated before, the main concern is the resource conservation because of the resource constraints of the sensor nodes hinders the usage of proven network protocols. The commonly huge amount of sensor nodes inhibits the reasonable deployment of a global addressing system, since the expense for ID maintenance would be to high [3]. The number of enclosed sensor nodes can reach up to 10.000 elements dependent on the required 
granularity of the measurement and robustness requisites [20].The sensor nodes on their own are not very reliable due to external ascendancies what leads to a varying quantity of sensor nodes. If nodes are added or die, this can have significant impact on communication paths and structure of the network. Time is a critical factor, not only since the data becomes eventually useless if the latency of the network is to high, but the transmitted data has to cover different numbers of hops respectively depending on the position of the sensor nodes varying distances.

\section{ENERGY DISSIPATION AT SOURCE END}

\subsection{Collisions}

If nodes receive multiple data packets at the same time collision occurs. In consequence the received data is useless and the transmission process has to be repeated while energy is dissipated. These re-transmissions can consume quite a lot of energy, since the energy losses are multiplied by the number of hops between source and target [11]. For reran transfers, methods like random delays can impede further collisions [2].

\subsection{Overhearing}

In high density sensor networks the short distances between sensor nodes lead to interferences with non-participant neighbour nodes during data conveyance. This impact on adjacent nodes is called Overhearing. As the transmitted signals idealised move circular from the sending signal source to its surrounding nodes, sensor nodes within reach, which are at the time in active mode, are a common problem. The not involved sensor nodes within reach burn up energy resources owing to receiving and processing useless information. Connectivity requirements have to be weighed up with the arising disadvantages regarding energy dissipation and latency caused by generously keeping nodes in active mode.

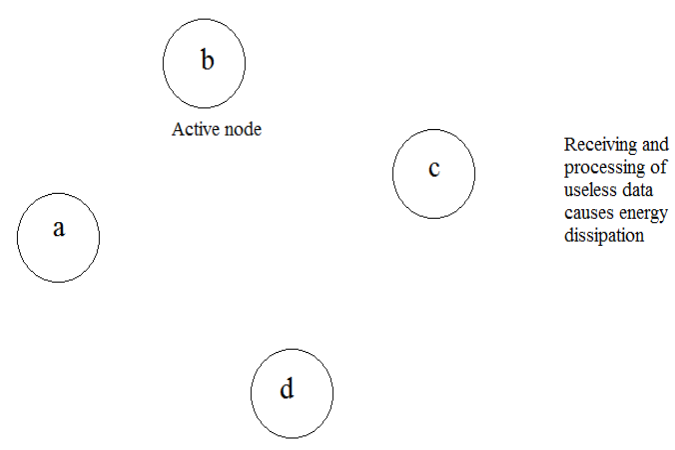

Fig 2: Overhearing

\subsection{Over Emitting}

As Overhearing data meant for other sensor nodes causes energy dissipation, so does Overemitting, meaning information propagated during inactive phases of the data sink or as the case may be the target node, which subsequently has to be resent [4]. The repeated transmissions increase the energy expenditures of the individual nodes for sending data and the latency of the whole sensor network. No customary but thoroughly imaginable defect is the simultaneous occurrence of Overhearing and Overemitting. The ideal situation would be a previously known path through the network with timely activation of the included sensor nodes, which in turn could lead to problems when concurrent transmissions take place and in unison paths are crossing.

\subsection{Idle Listening}

It is crucial to reduce the wasted energy resources, because only being in active mode is a major source of energy consumption and the difference of the expenditures for sending, receiving and waiting for transmission is relatively small. Usually the individual sensor node is not at any time involved in a data transmission process and thereby not every component of the node especially the Transceiver does implicitly have to be in an active state. This awaiting ready to transmit data while not receiving or sending packets is called idle listening. There are different approaches to find out when the particular components are not needed to be in active state or just reduce the overall active time.

\subsection{Traffic Fluctuation}

Traffic peaks caused by the event-based communication in Wireless Sensor Networks can temporarily lead to congestion or high delays [11]. When the network is working on its maximum capacity congestion rises to extremely high levels.

\subsection{Reduction of Protocol Overhead}

The transmission of e.g. protocol header information and control messages depletes energy resources and since this data in the end is not exploitable, it should be kept a minor share. Techniques for the reduction of the protocol overhead are for instance adaptive transmission periods, cross-layering approaches, where information from the other network layers is used for optimization, and optimized flooding to e.g. enable a less resource-intensive determination of locations[18]. A short transmission period leads to less energy consumption and helps therefore saving resources, at the same time latency to changes is increased [18]. In consequence a favourable value for the transmission period depends on the frequency of change.

\section{ENERGY CONSERVATION MEASURES}

\subsection{Node Activity Management}

\subsubsection{On-demand Node Activity}

In on-demand activity approaches the sleeping and transmission periods of the sensor nodes are not scheduled, but the nodes are by default permanently in an inactive state with a 
simple stand-by functionality. If a plain wake-up signal is broadcasted on a reserved channel, the neighboring nodes located within reach switch to active mode. After activation the data transmission can take place. As the start-up signal usually does not have to be decoded, a very energy conserving design for the listening device is feasible, nonetheless during transmission all surrounding nodes are switched on and for the most unnecessarily [4].

\subsubsection{Scheduled Rendezvous}

Scheduled time slots for simultaneous active phases of multiple adjacent sensor nodes can lead to a substantial simplification of the communication between the neighbouring nodes however for an effective application this method requires accurately synchronized internal timers [4].

\subsubsection{Sleep Scheduling}

Ready-to-receive mode consumes nearly as much energy of the sensor node's resources as receive mode [17]. Thus a way to set the node to a sleeping mode and determine the right time to wake it again is necessary to effectively save energy in idle time spans. The smaller the network load the greater are the saving potentials, as more nodes remain in active mode when they are not needed to. For sleep scheduling without any previous examination, certain periods are determined in which the individual node is in sleeping mode. The ideal time span for the periodically recurring inactive mode depends on the network traffic.

\subsection{WORKING ON DATA}

Working on data includes various operations such as Data aggregation, data fusion, data preprocessing, data reduction which are explained below.

\subsubsection{Data Compression}

Data compression saves energy by relocating effort from communication to processing. The sensing data is either previously gathered at some point or directly compressed to lower the workload for transmission. This leads to additional computational cost for compressing and decompressing, but at the same time information content is not reduced. Since processing data customarily consumes much less energy than transmitting data in a wireless medium the implementation of data compression algorithms can lead to considerable energy savings [10]. On the other hand the compression process takes time and hence increases latency of the sensor network. According to [10] the following categories of compression algorithms for Wireless Sensor Networks can be distinguished: Coding by ordering: The permutation of other variables within the sent data can be used to represent a value and therefore the overall sent data can be reduced. A permutation of three distinguishable variables can for example be utilized to save one of six different values. Pipelined in-network compression: The energy consumption is reduced by combining within a certain time span sensed data. Redundancies are removed and more data is aggregated into one data packet, which narrows the amount of sent data, but on the other side increases latency. Low-complexity video compression: Algorithm based on block changing detection and JPEG-compression for wireless video systems and especially designed to suit the limited hardware resources. Distributed compression: A side-information is used to encode information of two correlated sources and thereby to reduce the transmitted data.

\subsubsection{Deviation Control}

An alternative approach is to save the latest measured data and only report deviations which exceed a certain threshold. For this type of sensing the sensor nodes need a bu $\leftrightarrow$ er to save the latest values directly at the node and thereby reduce necessary data transmissions between the nodes. Dependent on the volatility of the sensed data the load of total network communication can significantly be reduced.

\subsubsection{Adaptive Sampling Rate}

The overall data can for example be reduced by adapting the sampling rate to the current situation. Circumstances permitting, this can significantly reduce the amount of generated data. Respective algorithms check for instance density in certain areas (the higher the density the lower the individual frequency), volatility of the sensed data or the traffic level to avoid congestion by lowering the sampling rates.

\subsubsection{Clustering}

Since the energy cost of transmitting data is higher than the effort on data processing it is beneficial to aggregate data within clusters [9]. Appointed sensor nodes which act as cluster heads provide the connection between sensor nodes and the respective base station [3]. Clustering can reduce the amount of data, as the cluster head is in charge of monitoring and processing queries [18] and thereby not as many connections have to be established. At meeting points of data from different sensor nodes redundancies can be recognized and avoided. Data aggregation is, due to the fact that it eases several of the energy dissipating effects, an important feature of energyefficient Wireless Sensor Networks, but one should way the extend, as buffering could lead to delays [11]. 


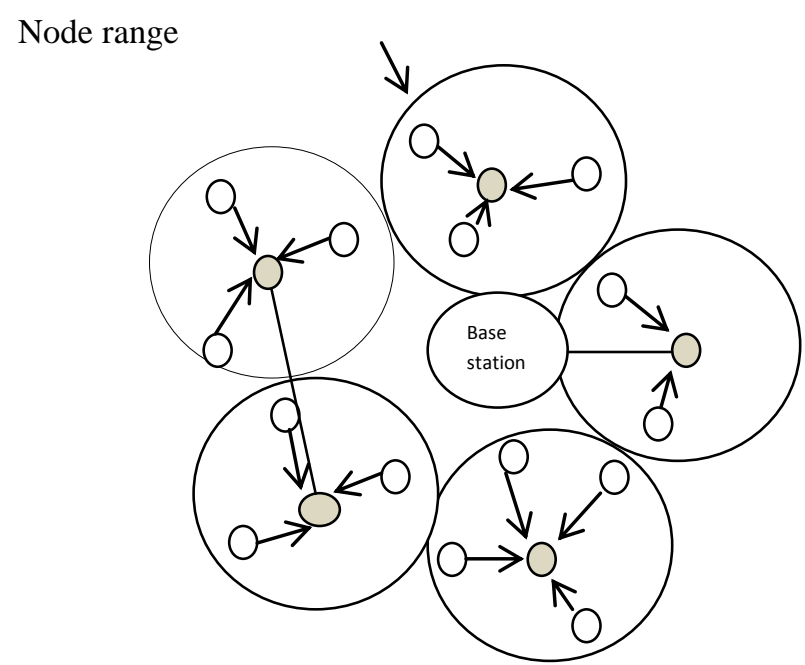

Fig 3: Clustering

\subsection{Load Balancing}

The actual position of a sensor node within the set of deployed nodes determines which operations have to be conducted and how many established communication paths comprise the particular node. The energy consumption can be balanced among the deployed sensor nodes e.g. by counting performed operations or by consideration of the remaining battery capacities. Due to the additional strain, regular sensor nodes appointed as cluster heads would have a much shorter lifetime as the nodes which are mere in charge of sensing data. If clustering is applied and there is no special more powerful hardware for the cluster heads in use, the usual approach for cluster head selection are stochastic methods which do not consider energy consumption [9]. As energy expenses of the cluster heads for data transmission are dependent on the distance between sensor node and base station, routing protocols like the in [13] proposed modification of LEACH, which consider cluster head energy conservation, have a positive effect on network lifetime, as they can divide the energy consumption into equal shares for all qualified nodes.

\subsection{Adaptive Transmission Range}

Since a reduction of the sensor node's broadcasting range lowers energy expenditure, the coverage should be broad enough to ensure proper connectivity, but all at once not unnecessarily extensive. Consideration of the remaining energy in addition to inclusion of the node distances obtains especially in heterogeneous sensor networks good results [18].

\subsection{Directional Antennas}

With directional antennas, as their name implies, all communication efforts are concentrated into a certain direction. This wages on the one hand additional problems, since the nodes for successful transmission not only have to be active but also properly oriented, on the other hand communication can take place over larger distances with less energy consumption and causes immediately less useless data which, on its way o the targeted sink, could lead to e. g. Overhearing and therefore more energy dissipation.

\section{COMMUNICATION PROTOCOL STACK}

\subsection{MAC Protocols}

Medium Access Control protocols for Wireless Sensor Networks can be categorized into centralized and distributed protocols with for each case the subgroups schedule-based and contention-based protocols, and hybrid protocols which combine the stated approaches [4]. Whereas the MAC protocol manages operations of the transducers and thus of the most energy consuming components of the sensor nodes, it is crucial for the energy-efficient implementation of a Wireless Sensor Network [6].

\subsubsection{Contention-Based Protocols}

Contention-based approaches are often associated with the use of Carrier Sense Multiple Access (CSMA) protocol, which is typically deployed in unsynchronized wireless networks, or a amended version of it [11]. CSMA examines the operating status of the channel before sending data and whenever it is occupied, transmissions are postponed [20]. If the medium is occupied, a random time interval is bided until the next status check is carried out. Otherwise the data transmission begins. For minor work loads contention-based protocols lead to higher channel utilization and are therefore in this case the means of choice. [19] states CSMA as the predominant class of Medium Access Control protocols. Representatives of these type are for example: B-MAC (Berkeley MAC): B-MAC is a very common MAC protocol as it is included in the TinyOS operating system. It offers a optimized carrier sense procedure and sleep interval definition within runtime [12]. S-MAC (Sensor-MAC) [5]: SMAC is a scheduled rendezvous approach with synchronization via sync packets. In listen periods the synchronization and other control information is exchanged. The nodes establish their own schedule and aggregate in virtual clusters. Contention-based protocols are robust, scalable, can easily adapt to traffic changes and facilitate low latency [5]. The downside is, that they are less energy efficient than TDMA based protocols.

\subsubsection{TDMA-Based Protocols}

TDMA (Time Division Multiple Access)-based approaches schedule the access to the medium by means of time slots. Time is partitioned in periodic frames with a certain number of slots and the channel access is managed on a slot by slot basis [5]. Since the delays are controlled and these contention-free protocols reduce or even eliminate collisions, high performance and reliability are consequent characteristics[20],[6]. TDMA is particularly suitable for high-traffic networks. Examples are the TRAMA (Traffic-Adaptive Medium access) protocol, which 
separates a random-access period with slot reservation and a scheduled-access period whose slots are assigned to individual nodes, and the for periodic monitoring applications optimized FLAMA (Flow-Aware Medium Access) protocol, which is based on TRAMA [5]. TDMA based protocols allow, provided the respective parameters are adequately appointed, a minimized energy consumption, but this precondition leads to limitations in adaptability to topology changes. These protocols are particularly qualified for high levels of contention and not as popular as the Contention-based type.

\subsubsection{Hybrid Protocols}

Hybrid Protocols change their behaviour dependent on the level of contention, which means they switch from a contention based behaviour at low contention levels to a TDMA approach in case of high levels of contention [5]. A established exponent of this category is Z-MAC (Zebra MAC), which employs CSMA in cases of slight traffic and otherwise avails TDMA [18]. Within a two-hop reach the sensor node, which wants to send data, has to contend for access. If a too many packets are lost, a control message is sent, which tells the neighbouring nodes to switch to high contention mode and after a certain time interval the nodes switch back to normal mode[12]. Even though the combination of the TDMA-based and the Contention-based approach can cancel out or at least alleviate the weaknesses of both protocol categories, it leads to high complexity and is arising thereby not practical for Wireless Sensor Networks with large node counts [5].

\subsection{Routing Protocols}

As consumed energy for transmission rises exponentially with distance [20], the sensing data is forwarded by directly neighbouring sensor nodes and the routing protocol has to specify the most suitable stopovers in terms of factors like load balancing and duration of the transfer. Besides classification by network structure (as conducted hereafter) routing protocols can be distinguished by their route establishment [11]. As proactive routing protocols on the one hand try to establish routes before they are actually needed and reactive protocols only react to inquiries, hybrid approaches combine the principles [11].

\subsubsection{Location-Based Routing}

Location-based routing's greatest advantage is the scalability. Due to the availability of location information for the sensor nodes, there is no global knowledge of the network necessary and thus routes can be specified and locations can be found independently from flooding [18]. Since location information is directly available, location-based protocols can help saving time and energy. The route determination is more efficient and apart from that the geographical information at hand can be used to only set the nodes of a specific area, which is of interest, in active mode, while the residual sensor nodes are in sleeping state [8]. GEAR (Geographic and Energy Aware
Routing): The respective neighbouring node and next hop to pass the data packet on is selected dependent on remaining energy resources and the distance to the destination [7]. MECN (Minimum Energy Communication Network) employs low power GPS and identifies relay regions for the respective nodes, inclosing the sensor nodes, which would selected as next hop lead to less energy consumption than a direct communication with the data sink [1].

\subsubsection{Hierarchical Routing}

Hierarchical routing protocols either subdivide the sensor nodes into clusters or appoint superior cluster heads which gather data originated by the cluster's nodes, process it and directly communicate with the sink or form other node sets to aggregate data and narrow the energy expenditures on data transmissions. Hierarchical routing enhances scalability and reduces the comprehensive traffic [18], but concurrently local energy cost for communication is increased due to e.g. the direct connection to the sink and the processing overhead [8]. Examples for hierarchical routing protocols are:

*LEACH (Low-Energy Adaptive Clustering Hierarchy) is a very popular hierarchical routing protocol which facilitates random change of cluster-heads to evenly balance the energy consumption and all data processing is carried out within the individual cluster [7].

*PEGASIS (Power-Efficient Gathering in Sensor Information Systems) instead of clusters forms node chains, which means that it is based on a multiple-hop strategy, and since there is no dynamic cluster formation necessary and the data aggregation reduces the number of transmissions, PEGASIS generally is more efficient than LEACH [1].

*TEEN (Threshold sensitive Energy Efficient sensor Network) is due to its responsiveness especially suitable for time-critical applications and the level of energy consumption is relatively low, as the sensor nodes only transmit values which exceed a certain predefined threshold[1].

\subsubsection{Data-Centric Routing}

For Wireless Sensor Networks with high numbers of nodes it is often not practical to assign global identifiers to every single node and as a result it is difficult to communicate with a particular node set [1]. As queries within the sensor network would otherwise lead to significant redundancies specific routing protocols to cope with this situation are necessary. In data-centric routing no great store is set by the originator of the transmitted data, so that the data easily can be combined and processed. Special cases of data centric routing are negotiationbased routing, where meta descriptions of the content are generated for advertising and interested nodes can request the data, and query-based routing, which is based on in-network processing by means of a pilot node aggregating the data and an ancillary query layer enabling inquiries of specific information [8]. Examples for data-centric routing protocols are: Flooding and Gossiping are antiquated mechanisms for broadcasting within a Wireless Sensor Network. For Flooding 
each node which receives data passes it on to its neighbouring nodes until a maximum number of hops are reached [7]. Gossiping is based on Flooding but the individual node which receives data relays it only to a randomly selected neighbour [7]. Common problems are duplicated messages sent to the same node (implosion) and two nodes sensing the same region sending similar data (overlap) [1].

*SPIN (Sensor Protocols for Information via Negotiation): Is based on negotiation between the nodes by means of data advertisement through meta-data. SPIN is one of the first datacentric routing protocols for Wireless Sensor Networks and it avoids typical flooding problems like overlaps, implosions and resource-blindness [7].

*For gradient-based routing the minimal number of intermediate stops on the way to the destination of the data is crucial. This minimum of waypoints is called height of the node [17]. The gap between the sensor node's height and the height of a neighbouring node represents the gradient of this particular connection. The largest difference between heights leads the way to the next hop.

\subsubsection{Quality-of-Service Based}

Routing Network-flow-based routing protocols resemble the already mentioned types but their main focus is to optimize the balancing of traffic and to maximize the network lifetime. Maximum lifetime energy routing for instance defines link costs depending on remaining energy and required transmission energy, which are utilized to even out the energy expenditures of the nodes [1]. Quality-of-Service functions like end-to-end guarantees and hence further examination of e.g. correct transmission are usually an additional feature of routing protocols. An example for a Quality-of-Service approach is the location-based protocol SPEED, which allows the estimation of end-to-end delays by ensuring a certain packet speed [1].

\subsection{Transport Protocols}

Transport protocol functionality is in several cases directly integrated in the routing protocol, though if implemented the transport protocol verifies the arrival of sent data at the sink which regulates the data traffic within the network and splits larger data loads into conveyable fragments [20]. There are three types of transport protocols [21]:

\subsubsection{Protocols for Reliability}

Protocols for reliability usually provide hop-to-hop loss recovery and therewith packet reliability. For loss detection and notification mostly NACK or IACK are used and popular types are for example RMSC, RBC and GARUDA [21]. An exception to the rule is ESRT, which provides event reliability by means of end-to-end source rate adjustment [21]. Since the connectivity within the Wireless Sensor Network is not predictable and the error rates for transmission are generally high, hop-to-hop loss recovery seems like the more adequate choice for WSNs. On the other hand an examination after every hop increases the latency. Reliability protocols can be distinguished by their reliability direction: Upstream (sensor to sink), downstream (sink to sensor) and bidirectional reliability [14].

\subsubsection{Protocols for Congestion Control}

Congestion is detected for instance via the queue length (Fusion, CODA) or packet service time (CCF, PCCP) and usually either implicitly or explicitly notified [21]. In most cases this notification is followed by a rate adjustment to mitigate the congestion. Exceptions are for example Siphon, which detects mitigation through queue length and application fidelity and reacts by redirecting traffic to virtual sinks, or Trickle, which aims to prevent congestion by utilising a politegossip approach and there needs no additional congestion detection [21].

\subsubsection{Protocols for Congestion Control and Reliability}

Transport protocols like STCP combine the two previous types and provide congestion control as well as reliability [21]. Other examples are RCRT, CRRT and TRCCIT [14].

\section{CONCLUSIONS}

As there is a tremendous decrease in the cost of wireless sensor network's node and its equipments and also in advancing the network lifetime of a wireless sensor networks, which results into a raise in number of potential application area both energy conservation and communication techniques become increasingly important and has become research projects. As we know most of the sensor nodes consumes energy at communication more than computation. The consumption is not just in transmission state but as well in idle state, relatively high and depending on the application, the sensing unit can spend considerable amount of energy. TDMA based MAC protocol are very energy efficient, if correctly configured, contention based approaches on the other hand are very robust and flexible by means of traffic changes and scalability. With this integration in hybrid protocol and thereby a combination of their strength is desirable, but understanding the complexity, which is not suitable for wireless sensor network's with high volume of nodes. The basic evolution shows that the best results are obtained with combination of the termed energy saving measures. The most suitable solution for certain applications depends upon various factors such as environment, size of network, types of a data, hardware components and change in frequency.

\section{REFERENCES}

[1] K. Akkaya and M. Younis. A survey on routing protocols for wireless sensor networks. Ad hoc networks, 3(3):325-349, 2005.

[2] I. Akyildiz, W. Su, Y. Sankarasubramaniam, and E. Cayirci. A survey on sensor networks. 
Communications Magazine, IEEE, 40(8):102 - 114, aug 2002.

[3] J. Al-Karaki and A. Kamal. Routing techniques in wireless sensor networks: a survey. Wireless Communications, IEEE, 11(6):6 - 28, dec. 2004.

[4] M. A. Ameen, S. M. R. Islam, and K. Kwak. Energy saving mechanisms for mac protocols in wireless sensor networks. International Journal of Distributed Sensor Networks, 2010, 2010.

[5] G. Anastasi, M. Conti, M. D. Francesco, and A. Passarella. Energy conservation in wireless sensor networks: A survey. Ad Hoc Networks, 7(3):537 $568,2009$.

[6] C. Cano, B. Bellalta, A. Sfairopoulou, and M. Oliver.Low energy operation in wsns: A survey of preamble sampling mac protocols. Computer Networks, 55(15):3351 - 3363, 2011.

[7] S. Dai, X. Jing, and L. Li. Research and analysis on routing protocols for wireless sensor networks. In Communications, Circuits and Systems, 2005. Proceedings. 2005 International Conference on, volume 1, pages 407-411. IEEE, 2005.

[8] S. Ehsan and B. Hamdaoui. A survey on energye_cient routing techniques with qos assurances for wireless multimedia sensor networks. Communications Surveys Tutorials, IEEE, 14(2):265278, quarter 2012.

[9] W. Heinzelman, A. Chandrakasan, and $\mathrm{H}$. Balakrishnan. An application-specific protocol architecture for wireless microsensor networks.Wireless Communications, IEEE Transactions on, 1(4):660 - 670, oct 2002.

[10] N. Kimura and S. Latifi. A survey on data Compression in wireless sensor networks. In Information Technology: Coding and Computing, 2005. ITCC 2005. International Conference on, volume 2, pages 8-13. Ieee, 2005.

[11] A. Klein. Performance Issues of MAC and Routing Protocols in Wireless Sensor Networks. PhD thesis, University of W“urzburg, Dec. 2010.

[12] K. Langendoen. Medium access control in wireless sensor networks. Medium access control in wireless networks, 2:535-560, 2008.

[13] N. V. Lonbale, S. Gupta, and R. S. Bhute. Energy conservation of wsn using routing protocol. IJCA Proceedings on National Conference on Innovative Paradigms in Engineering and Technology (NCIPET 2012), ncipet(5):18-21, March 2012. Published by Foundation of Computer Science, New York, USA.

[14] A. Rathnayaka, V. Potdar, A. Sharif, S. Sarencheh, and S. Kuruppu. Wireless sensor networks: Challenges ahead. In Broadband, Wireless Computing, Communication and Applications (BWCCA), 2010 International Conference on, pages 824-829. IEEE, 2010 .
[15] Z. Ren, S. Shi, Q. Wang, and Y. Yao. A node sleeping algorithm for wsns based on the minimum hop routing protocol. In Computer and Management (CAMAN), 2011 International Conference on, pages 1 -4 , may 2011.

[16] I. Rhee, A. Warrier, M. Aia, J. Min, and M. Sichitiu. Z-mac: a hybrid mac for wireless sensor networks. IEEE/ACM Transactions on Networking (TON), 16(3):511-524, 2008.

[17] J. Sinha and S. Barman. Energy efficient routing mechanism in wireless sensor network. In Recent Advances in Information Technology (RAIT), $20121^{\text {st }}$ International Conference on, pages $300-305$, march 2012.

[18] R. Soua and P. Minet. A survey on energy e_cient techniques in wireless sensor networks. In Wireless and Mobile Networking Conference (WMNC), 2011 4th Joint IFIP, pages $1-9$, oct. 2011.

[19] J. Stankovic and T. He. Energy management in sensor networks. Philosophical Transactions of the Royal Society A: Mathematical, Physical and Engineering Sciences, 370(1958):52-67, 2012.

[20] J. Suhonen, M. Kohvakka, V. Kaseva, T. D. H“am"al"ainen, and M. H“annik"ainen. Low-Power Wireless Sensor Networks - Protocols, Services and Applications. Springer, Berlin, Heidelberg, 2012. aufl. edition, 2012.

[21] C. Wang, K. Sohraby, B. Li, M. Daneshmand, and Y. Hu. A survey of transport protocols for wireless sensor networks. Network, IEEE, 20(3):34-40, 2006. 